\title{
The Integral Representations of Harmonic Polynomials in the Case of $\mathfrak{s p}(p, 1)$
}

\author{
Ryoko WADA
}

Kure University

(Communicated by T. Nagano)

\section{Introduction.}

Let $\mathfrak{g}$ be a complex reductive Lie algebra and let $\mathfrak{g}=\mathfrak{f}+\mathfrak{p}$ be the complexification of a Cartan decomposition of $\mathfrak{g}_{\mathbf{R}}=\mathfrak{f}_{\mathbf{R}}+\mathfrak{p}_{\mathbf{R}}$, where $\mathfrak{g}_{\mathbf{R}}$ is a noncompact real form of $\mathfrak{g}$.

Kostant-Rallis [3] stated some results on harmonic polynomials on $\mathfrak{p}$. On the other hand, for classical harmonic polynomials on $\mathbf{C}^{p}$, there have been many studies. It is well known that harmonic functions on $\mathbf{C}^{p}$ are represented by integrals on the unit sphere $S^{p-1}$ or on some other $O(p)$-orbits and reproducing kernels of these formulas are expressed by Legendre polynomials (see, [2], [4], [5], [6], [7], [10], [12], [15], etc.).

In our previous paper [14] we obtained explicit integral representation formulas of harmonic polynomials in the case $\mathfrak{g}_{\mathbf{R}}=\mathfrak{s u}(p, 1)$. From the Lie algebraic viewpoint, classical harmonic functions on $\mathbf{C}^{p}$ corresponds to harmonic functions on $\mathfrak{p}$ for the case $\mathfrak{g}_{\mathbf{R}}=\mathfrak{s \mathfrak { D }}(p, 1)$. Therefore, integral representation formulas of harmonic polynomials in this case are known.

Our purpose of this paper is to obtain explicit integral representation formulas of harmonic polynomials and reproducing kernel of these formulas in remaining classical real rank one case, i.e. the case $\mathfrak{g}_{\mathbf{R}}=\mathfrak{s p}(p, 1)$. Our main results in this paper are described in Theorem 2.2, in which harmonic polynomials on $\mathfrak{p}$ for $\mathfrak{g}_{\mathbf{R}}=\mathfrak{s p}(p, 1)$ are represented by an integral on some $K_{\mathbf{R}}$-orbits. This result is similar to the cases $\mathfrak{g}_{\mathbf{R}}=\mathfrak{s o}(p, 1)$ and $\mathfrak{g}_{\mathbf{R}}=\mathfrak{s u}(p, 1)$.

The author would like to thank Professor M. Morimoto and Professor Y. Agaoka for their helpful advices.

\section{Preliminaries.}

In this section we fix notations and review known results. For details, see [2], [3], [5], [7] and [16].

Received November 18, 1997 
Let $\mathfrak{g}$ be a complex reductive Lie algebra and let $\mathfrak{g}_{R}$ be a noncompact real form of g. Let $\mathfrak{g}_{\mathbf{R}}=\mathfrak{f}_{\mathbf{R}}+\mathfrak{p}_{\mathbf{R}}$ be a Cartan decomposition of $\mathfrak{g}_{\mathbb{R}}$ and let $\mathfrak{g}=\mathfrak{f}+\mathfrak{p}$ be the direct sum obtained by complexifying $\mathfrak{f}_{\mathbf{R}}$ and $\mathfrak{p}_{\mathbf{R}}$. In this paper, for a Lie algebra $\mathfrak{h}$, we denote by expadh the adjoint group of $\mathfrak{h}$. We put $G=\exp$ adg and $K_{\theta}=\{a \in G ; \theta a=a \theta\}$, where $\theta: \mathfrak{g} \rightarrow \mathfrak{g}$ is the Lie algebra automorphism of order 2 defined by $\theta=1$ on $\mathfrak{f}, \theta=-1$ on p. Let $K$ be the identity component of $K_{\theta}$. Then we have $K=\operatorname{expadf}$. Furthermore, we put $K_{R}=\operatorname{expad\mathfrak {f}_{R}}$, which acts on the space $\mathfrak{p}$. Then we have $K_{\mathbf{R}}=K \cap \operatorname{expadg_{R}}$. We denote by $S$ the symmetric algebra on $p$. We put $J=\left\{u \in S ; a u=u\right.$ for any $\left.a \in K_{\theta}\right\}$ and $J_{+}=\{u \in J ; \partial(u) 1=0\}$. We denote by $J^{\prime}$ the ring of $K$-invariant polynomials on $\mathfrak{p}$ and we put $J_{+}^{\prime}=\left\{f \in J^{\prime} ; f(0)=0\right\}$. Let $S^{\prime}$ be the ring of all polynomials on $\mathfrak{p}$ and let $S_{n}^{\prime}$ be the space of homogeneous polynomials on $\mathfrak{p}$ of degree $n$. For $f \in S^{\prime}$ and $a \in K_{\theta}$, af $\in S^{\prime}$ is defined by $(a f)(x)=f\left(a^{-1} x\right)(x \in \mathfrak{p})$. It is known that any element of $J^{\prime}$ is invariant under $K_{\theta}$ ([3] Proposition 10). It is also known that $J^{\prime}$ has homogeneous generators $P_{1}, \cdots, P_{r}$, where $r=\operatorname{dim} a_{R}$ and $a_{R}$ is a maximal abelian subalgebra of $\mathfrak{p}_{\mathbf{R}} \cdot \operatorname{dim} \mathfrak{a}_{\mathbf{R}}$ is called the real rank of $\mathfrak{g}_{\mathbf{R}}$. Let $\mathscr{H}=\left\{f \in S^{\prime} ; \partial(u) f=0\right.$ for any $\left.u \in J_{+}\right\}$be the space of harmonic polynomials on $p$. We put $\mathscr{H}_{n}=S_{n}^{\prime} \cap \mathscr{H}$ and $J_{n}^{\prime}=S_{n}^{\prime} \cap J^{\prime}$. We put $\left(J_{+}^{\prime} S^{\prime}\right)_{n}=J_{+}^{\prime} S^{\prime} \cap S_{n}^{\prime}$, and $\mathbf{Z}_{+}=\{0,1,2,3, \cdots\}$. It is known that $S_{n}^{\prime}=\left(J_{+}^{\prime} S^{\prime}\right)_{n} \oplus \mathscr{H}_{n}\left(n \in \mathbf{Z}_{+}\right)$ (see [3]). We put $\mathfrak{N}=\left\{X \in \mathfrak{p} ; P(X)=0\right.$ for any $\left.P \in J_{+}^{\prime}\right\}$. It is known that the restriction mapping $\left.f \rightarrow f\right|_{\mathfrak{N}}$ is a bijection from $\mathscr{H}$ onto $\left.\mathscr{H}\right|_{\mathfrak{N}}$ (see [8], [14]).

$H_{n}\left(C^{q}\right)$ denotes the space of homogeneous harmonic polynomials of degree $n$ on $\mathrm{C}^{q}$ and $H_{n, q}$ denotes the space of spherical harmonics of degree $n$ on $S^{q-1}$. It is well known that

$$
\operatorname{dim} H_{n, q}=\frac{(2 n+q-2)(n+q-3) !}{n !(q-2) !} .
$$

It is well known that the restriction mapping $\left.f \rightarrow f\right|_{S^{q-1}}$ is a bijection from $H_{n}\left(\mathbf{C}^{q}\right)$ onto $H_{n, q}$. For spherical harmonics and harmonic functions on $\mathbf{C}^{q}$, we refer the reader to [5], [6], [7], etc.

$P_{n, q}$ denotes the Legendre polynomial of degree $n$ and dimension $q$. For $z, w \in \mathbf{C}^{q}$ we put $z \cdot w={ }^{t} z w$. We put $N^{q-1}=\left\{z \in \mathbf{C}^{q} ; z \cdot z=0, z \cdot \bar{z}=2\right\}$. Then the following lemma is known.

LEMMA 1.1 ([2], [5], [6], [7], [16]). (i) We put $h_{a}(x)=P_{n, q}(x \cdot a)$ and $g_{b}(x)=(x \cdot b)^{n}$ $\left(x, a, b \in C^{q}\right)$. Then $H_{n, q}$ is generated by the set $\left\{\left.h_{a}\right|_{S^{q-1}} ; a \in S^{q-1}\right\}$ and $H_{n}\left(C^{q}\right)$ is generated by the set $\left\{g_{b} ; b \in N^{q-1}\right\}$.

(ii) For any $f \in H_{m}\left(\mathbf{C}^{q}\right)$ and $g \in H_{n}\left(\mathbf{C}^{q}\right)$ it is valid that

$$
\begin{array}{r}
\operatorname{dim} H_{n, q} \int_{S^{q-1}} f(s) P_{n, q}(s \cdot a) d s=\delta_{m, n} f(a) \quad\left(a \in S^{q-1}\right), \\
\operatorname{dim} H_{n, q} \int_{N^{q-1}} f(z)(\bar{z} \cdot w)^{n} d N(z)=2^{n} \delta_{m, n} f(w) \quad\left(w \in \mathbf{C}^{q}\right),
\end{array}
$$




$$
\int_{S^{q-1}} f(s) \overline{g(s)} d s=2^{-2 n} \frac{n ! \Gamma(q / 2)}{\Gamma(n+q / 2)} \delta_{m, n} \operatorname{dim} H_{n, q} \int_{N^{q-1}} f(z) \overline{g(z)} d N(z),
$$

where $d s$ and $d N$ denote the unique $O(q)$-invariant measures on $S^{q-1}$ and on $N^{q-1}$ such that $\int_{S q-1} 1 d s=\int_{N^{q-1}} 1 d N(z)=1$, respectively.

For $z \in \mathbf{C}^{q}$ and $a \in S^{q-1}$ we put

$$
\tilde{P}_{n, q}(z, a)=P_{n, q}\left(\frac{z \cdot a}{\sqrt{z \cdot z}}\right)(z \cdot z)^{n / 2} .
$$

Then $\widetilde{P}_{n, q}(, a)$ belongs to $H_{n}\left(C^{q}\right)$ and $\widetilde{P}_{n, q}(s, a)=P_{n, q}(s \cdot a)$ for any $s \in S^{q-1}$.

\section{The case $\mathfrak{g}_{\mathbf{R}}=\mathfrak{s p}(p, 1)$.}

In this section we obtain integral representation formulas of harmonic polynomials on some $K_{\mathbf{R}}$-orbits in the case $\mathfrak{g}_{\mathbf{R}}=\mathfrak{s p}(p, 1)\left(p \in \mathbf{Z}_{+}, p \geq 2\right)$. We put

$$
\begin{aligned}
& \mathfrak{g}=\mathfrak{s p}(p+1, \mathbf{C})=\left\{\left(\begin{array}{cc}
A & B \\
C & -{ }^{t} A
\end{array}\right) ; A, B, C \in M(p+1, \mathbf{C}),{ }^{t} B=B,{ }^{t} C=C\right\}, \\
& \mathfrak{g}_{\mathbf{R}}=\mathfrak{s p}(p, 1)=\left\{\left(\begin{array}{cccc}
A & x & B & y \\
{ }^{t} \bar{x} & a & { }^{t} y & b \\
-\bar{B} & \bar{y} & \bar{A} & -\bar{x} \\
{ }^{t} \bar{y} & -\bar{b} & -{ }^{t} x & \bar{a}
\end{array}\right) ; \begin{array}{l}
A \in \mathfrak{u}(p), a \in \mathfrak{u}(1) \\
B \text { is } p \times p \text { symmetric } \\
b \in \mathbf{C}, x, y \in \mathbf{C}^{p}
\end{array}\right\}, \\
& \mathfrak{f}_{\mathbf{R}}=\left\{\left(\begin{array}{cccc}
A & 0 & B & 0 \\
0 & a & 0 & b \\
-\bar{B} & 0 & \bar{A} & 0 \\
0 & -\bar{b} & 0 & \bar{a}
\end{array}\right) ; \begin{array}{l}
A \in \mathfrak{u}(p), a \in \mathfrak{u}(1), b \in \mathbf{C} \\
B \text { is } p \times p \text { symmetric }
\end{array}\right\}, \\
& \mathfrak{p}_{\mathbf{R}}=\left\{\left(\begin{array}{cccc}
0 & x & 0 & y \\
{ }^{t} \bar{x} & 0 & { }^{t} y & 0 \\
0 & \bar{y} & 0 & -\bar{x} \\
{ }^{t} \bar{y} & 0 & -{ }^{t} x & 0
\end{array}\right) ; x, y \in \mathbf{C}^{p}\right\} \text {. }
\end{aligned}
$$

Then we have

$$
\mathfrak{f}=\left\{\left(\begin{array}{cccc}
A & 0 & B & 0 \\
0 & \alpha & 0 & \beta \\
C & 0 & -{ }^{t} A & 0 \\
0 & \gamma & 0 & -\alpha
\end{array}\right), \begin{array}{l}
A, B, C \in M(p, \mathbf{C}) \\
{ }^{t} B=B, \beta, \gamma=C \\
\alpha, \beta, C
\end{array}\right\},
$$




$$
\mathfrak{p}=\left\{\left(\begin{array}{cccc}
0 & x & 0 & w \\
{ }^{t} y & 0 & { }^{t} w & 0 \\
0 & z & 0 & -y \\
{ }^{t} z & 0 & -{ }^{t} x & 0
\end{array}\right) ; x, y, z, w \in \mathbf{C}^{p}\right\},
$$

$G=\operatorname{Ad} \operatorname{Sp}(p+1, \mathbf{C})$,

$$
\begin{aligned}
& K=\left\{\operatorname{Ad}\left(\begin{array}{cccc}
A & 0 & B & 0 \\
0 & \alpha & 0 & \beta \\
C & 0 & D & 0 \\
0 & \gamma & 0 & \delta
\end{array}\right) ; \begin{array}{l}
A, B, C, D \in M(p, \mathbf{C}), \alpha, \beta, \gamma, \delta \in \mathbf{C} \\
{ }^{t} C A={ }^{t} A C,{ }^{t} D B={ }^{t} C B D=I_{p}, \alpha \delta-\beta \gamma=1
\end{array}\right\},
\end{aligned}
$$

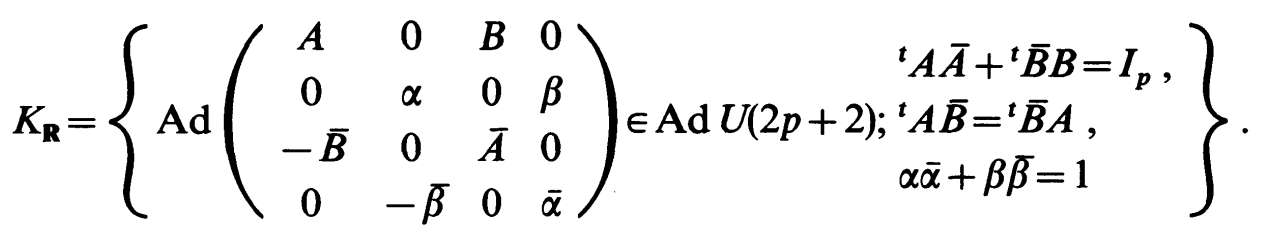

For this Lie algebra $g$ the Killing form $B(X, Y)$ equals $(2 p+4) \operatorname{Tr}(X Y)$. The generator of $J^{\prime}$ is $B(X, X)(X \in \mathfrak{p})$. For $X=\left(\begin{array}{cccc}0 & x & 0 & w \\ t^{y} & 0 & { }^{t} w & 0 \\ 0 & z & 0 & -y \\ t_{z} & 0 & -{ }^{t} x & 0\end{array}\right) \in \mathfrak{p}$, we put $P(X)=4(x \cdot y+z \cdot w)=$ $\operatorname{Tr} X^{2}$. Then $\mathscr{H}_{n}=\left\{f \in S_{n}^{\prime} ; P(D) f(X)=0\right\}$, where $P(D)=4 \sum_{j=1}^{p}\left(\frac{\partial^{2}}{\partial x_{j} \partial y_{j}}+\frac{\partial^{2}}{\partial z_{j} \partial w_{j}}\right)$. Furthermore $\mathfrak{N}=\{X \in \mathfrak{p} ; B(X, X)=0\}=\{X \in \mathfrak{p} ; x \cdot y+z \cdot w=0\}$. We put

$$
\Sigma=\{X \in \mathfrak{p} ; y=\bar{x}, w=\bar{z}, x \cdot \bar{x}+z \cdot \bar{z}=1\} .
$$

Let $g=\operatorname{Ad}\left(\begin{array}{cccc}A & 0 & B & 0 \\ 0 & \alpha & 0 & \beta \\ -\bar{B} & 0 & \bar{A} & 0 \\ 0 & -\bar{\beta} & 0 & \bar{\alpha}\end{array}\right) \in K_{\mathbf{R}}$. If we put $g X=\left(\begin{array}{cccc}0 & x^{\prime} & 0 & w^{\prime} \\ y^{\prime} y^{\prime} & 0 & { }^{t} w^{\prime} & 0 \\ 0 & z^{\prime} & 0 & -y^{\prime} \\ t_{z^{\prime}} & 0 & -{ }^{t} x^{\prime} & 0\end{array}\right) \in \mathfrak{p}$, we have

$$
\begin{aligned}
& x^{\prime}=A(\bar{\alpha} x+\bar{\beta} w)+B(\bar{\alpha} z-\bar{\beta} y), \\
& z^{\prime}=-\bar{B}(\bar{\alpha} x+\bar{\beta} w)+\bar{A}(\bar{\alpha} z-\bar{\beta} y), \\
& y^{\prime}=\bar{B}(-\beta x+\alpha w)+\bar{A}(\alpha y+\beta z), \\
& w^{\prime}=A(-\beta x+\alpha w)-B(\alpha y+\beta z) .
\end{aligned}
$$

By putting $\Phi(X)=\left(\begin{array}{c}x \\ z \\ y \\ w\end{array}\right)$, we can rewrite (2.1) as follows:

$$
\Phi(g X)=\Lambda(g) \Phi(X)
$$

where 


$$
\Lambda(g)=\left(\begin{array}{cc}
\bar{\alpha} A_{1} & -\bar{\beta} A_{2} \\
-\beta \bar{A}_{2} & \alpha \bar{A}_{1}
\end{array}\right), \quad A_{1}=\left(\begin{array}{cc}
A & B \\
-\bar{B} & \bar{A}
\end{array}\right), \quad A_{2}=\left(\begin{array}{cc}
B & -A \\
\bar{A} & \bar{B}
\end{array}\right) .
$$

We see that $A_{1}, A_{2} \in U(2 p)$ and $\Lambda(g) \in U(4 p)$.

Next for $X \in \mathfrak{p}$ we define the mapping $\Psi: \mathfrak{p} \rightarrow C^{4 p}$ by

$$
\Psi(X)=\frac{1}{2}\left(\begin{array}{c}
x+y \\
z+w \\
-i(x-y) \\
-i(z-w)
\end{array}\right)
$$

It is clear that $\Psi$ is the bijection from $\Sigma$ onto $S^{4 p-1}$. We can see that $f \in \mathscr{H}_{n}$ if and only if $f \circ \Psi^{-1} \in H_{n, 4 p}$. Therefore, we have $\operatorname{dim} \mathscr{H}_{n}=\operatorname{dim} H_{n, 4 p}$. We put $\langle X, Y\rangle=$ $\frac{1}{2} \operatorname{Tr}\left({ }^{t} X \bar{Y}\right)=\Phi(X) \cdot \overline{\Phi(Y)}$ and $Q_{n, p}(X, Z)=\tilde{P}_{n, 4 p}(\Psi(X), \Psi(Z))(X, Y \in \mathfrak{p}, Z \in \Sigma)$. Then

$$
Q_{n, p}(X, Z)=2^{-n} P_{n, 4 p}\left(\frac{\langle X, Z\rangle}{\sqrt{P(X)}}\right)(P(X))^{n / 2} .
$$

$\mathscr{H}_{n}$ is generated by $\left\{Q_{n, p}(, Z) ; Z \in \Sigma\right\}$. It is also known that $\mathscr{H}_{n}$ is generated by $\left\{\langle, Z\rangle^{n} ; Z \in \mathfrak{N}\right\}$ (see [3]). For $g=\operatorname{Ad}\left(\begin{array}{cccc}A & 0 & B & 0 \\ 0 & \alpha & 0 & \beta \\ -\bar{B} & 0 & \bar{A} & 0 \\ 0 & -\bar{\beta} & 0 & \bar{\alpha}\end{array}\right) \in K_{\mathbf{R}}$ we get

$$
\Psi(g X)=M(g) \Psi(X)
$$

where

$$
M(g)=\left(\begin{array}{cc}
\operatorname{Re}\left(\bar{\alpha} A_{1}-\beta \bar{A}_{2}\right) & -\operatorname{Im}\left(\bar{\alpha} A_{1}-\beta \bar{A}_{2}\right) \\
\operatorname{Im}\left(\bar{\alpha} A_{1}+\beta \bar{A}_{2}\right) & \operatorname{Re}\left(\bar{\alpha} A_{1}+\beta \bar{A}_{2}\right)
\end{array}\right) .
$$

Note that $M(g) \in O(4 p)$. Let

$$
E_{0}=\left(\begin{array}{cccc}
0 & e_{1} & 0 & 0 \\
{ }^{t} e_{1} & 0 & 0 & 0 \\
0 & 0 & 0 & -e_{1} \\
0 & 0 & -{ }^{t} e_{1} & 0
\end{array}\right) \in \Sigma
$$

where $e_{1}={ }^{t}\left(\begin{array}{lllll}1 & 0 & 0 & \cdots & \cdots\end{array}\right) \in \mathbf{C}^{p}$. Then we have the following

LEMMA 2.1. It is valid that $\Sigma=K_{\mathbf{R}} E_{0}$.

Proof. From (2.1) and (2.2) it is easy to show that $K_{\mathrm{R}} E_{0} \subset \Sigma$ because $\Lambda(g)$ belongs to $U(4 p)$. Suppose $X=\Phi^{-1}\left(t^{t}(x z \bar{x} \bar{z})\right) \in \Sigma$. Then $\left(\begin{array}{c}x \\ z\end{array}\right)$ and $\left(\begin{array}{c}-\bar{z} \\ \bar{x}\end{array}\right)$ form an orthonormal 
system of $\mathbf{C}^{2 p}$. There is some $\left(\begin{array}{l}a_{2} \\ b_{2}\end{array}\right) \in \mathbf{C}^{2 p} \backslash\{0\}$ such that $\left(\begin{array}{c}x \\ z\end{array}\right),\left(\begin{array}{c}-\bar{z} \\ \bar{x}\end{array}\right)$ and $\left(\begin{array}{l}a_{2} \\ b_{2}\end{array}\right)$ form an orthonormal system of $\mathbf{C}^{2 p}$. Then we see that $\left(\begin{array}{c}x \\ z\end{array}\right),\left(\begin{array}{c}-\bar{z} \\ \bar{x}\end{array}\right),\left(\begin{array}{c}a_{2} \\ b_{2}\end{array}\right)$ and $\left(\begin{array}{c}-\bar{b}_{2} \\ \bar{a}_{2}\end{array}\right)$ form an orthonormal system of $\mathbf{C}^{2 p}$. Repeating this procedure, we can find $a_{j}, b_{j} \in \mathbf{C}^{p}$ $(j=2,3, \cdots, p)$ such that $\left\{\left(\begin{array}{c}x \\ z\end{array}\right),\left(\begin{array}{c}-\bar{z} \\ \bar{x}\end{array}\right),\left(\begin{array}{c}a_{j} \\ b_{j}\end{array}\right),\left(\begin{array}{c}-b_{j} \\ \bar{a}_{j}\end{array}\right)(2 \leq j \leq p)\right\}$ is an orthonormal basis of $\mathbf{C}^{2 p}$. If we put $A=\left(x a_{2} \cdots a_{p}\right)$ and $B=\left(-\bar{z}-\bar{b}_{2} \cdots-\bar{b}_{p}\right)$, we have ${ }^{t} A \bar{A}+{ }^{t} \bar{B} B=I_{p}$ and ${ }^{t} A \bar{B}={ }^{t} \bar{B} A$ because $\left(\begin{array}{cc}A & B \\ -\bar{B} & \bar{A}\end{array}\right) \in U(2 p)$. Therefore, by putting $g=\operatorname{Ad}\left(\begin{array}{cccc}A & 0 & B & 0 \\ 0 & 1 & 0 & 0 \\ -\bar{B} & 0 & \bar{A} & 0 \\ 0 & 0 & 0 & 1\end{array}\right)$, we get $g \in K_{\mathbf{R}}$ and $g E_{0}=X$. Hence we have $\Sigma=K_{\mathbf{R}} E_{0}$.

Q.E.D.

We denote by $K_{0}$ the isotropy group of $K_{\mathrm{R}}$ at $E_{0}$. Then we have

$$
K_{0}=\left\{\operatorname{Ad}\left(\begin{array}{cccccc}
\alpha & 0 & 0 & -\beta & 0 & 0 \\
0 & A^{\prime} & 0 & 0 & B^{\prime} & 0 \\
0 & 0 & \alpha & 0 & 0 & \beta \\
\bar{\beta} & 0 & 0 & \bar{\alpha} & 0 & 0 \\
0 & -\bar{B}^{\prime} & 0 & 0 & \bar{A}^{\prime} & 0 \\
0 & 0 & -\bar{\beta} & 0 & 0 & \bar{\alpha}
\end{array}\right) \in K_{\mathbf{R}} ; \begin{array}{c}
{ }^{t} A^{\prime} \bar{A}^{\prime} \bar{B}^{\prime}+{ }^{t} \bar{B}^{\prime} \bar{B}^{\prime} \bar{B}^{\prime} A^{\prime}, \\
\alpha \bar{\alpha}+\beta \bar{\beta}=1
\end{array}\right\} .
$$

Next we put $\tilde{E}_{r}=\Phi^{-1}\left(\begin{array}{c}r e_{1} \\ 0 \\ 0 \\ \left(1-r^{2}\right)^{1 / 2} e_{2}\end{array}\right) \in \mathfrak{N}$ and $N(r)=K_{\mathrm{R}} \tilde{E}_{r}, \quad(0 \leq r \leq 1)$, where $e_{2}={ }^{t}\left(\begin{array}{lll}0 & 1 & \cdots\end{array}\right) \in \mathbf{C}^{p}$. Let $d \mu_{\Sigma}$ and $d \mu_{r}$ be the unique $K_{\mathbf{R}}$-invariant measures on $\Sigma$ and $N(r)$ such that $\int_{\Sigma} 1 d \mu_{\Sigma}=\int_{N(r)} 1 d \mu_{r}=1$. Since $M(g) \in O(4 p)$, we get $\int_{\Sigma} f(Z) d \mu_{\Sigma}(Z)=$ $\int_{S^{4 p-1}} f \circ \Psi^{-1}(s) d s$. For $g=\operatorname{Ad}\left(\begin{array}{cccc}A & 0 & B & 0 \\ 0 & \alpha & 0 & \beta \\ -\bar{B} & 0 & \bar{A} & 0 \\ 0 & -\bar{\beta} & 0 & \bar{\alpha}\end{array}\right) \in K_{\mathbf{R}}$ we have

$$
\Phi\left(g \widetilde{E}_{r}\right)=\left(\begin{array}{c}
x \\
z \\
y \\
w
\end{array}\right),
$$

where 


$$
\begin{aligned}
& \left(\begin{array}{l}
x \\
z
\end{array}\right)=\left(\begin{array}{l}
\bar{\alpha} r a_{1}+\bar{\beta}\left(1-r^{2}\right)^{1 / 2} a_{2} \\
-\bar{\alpha} r \bar{b}_{1}-\bar{\beta}\left(1-r^{2}\right)^{1 / 2} \bar{b}_{2}
\end{array}\right), \\
& \left(\begin{array}{l}
y \\
w
\end{array}\right)=\left(\begin{array}{l}
-\beta r \bar{b}_{1}+\alpha\left(1-r^{2}\right)^{1 / 2} \bar{b}_{2} \\
-\beta r a_{1}+\alpha\left(1-r^{2}\right)^{1 / 2}{ }_{2} a_{2}
\end{array}\right),
\end{aligned}
$$

and $a_{j}=A e_{j}, b_{j}=B e_{j}(j=1,2)$. Now from the condition on $K_{\mathbf{R}}$ we have

$$
\left(\begin{array}{c}
a_{i} \\
-\bar{b}_{i}
\end{array}\right) \cdot\left(\begin{array}{c}
\bar{b}_{j} \\
a_{j}
\end{array}\right)=0, \quad\left(\begin{array}{c}
a_{i} \\
-\bar{b}_{i}
\end{array}\right) \cdot\left(\begin{array}{c}
\bar{a}_{j} \\
-b_{j}
\end{array}\right)=\delta_{i, j} \quad(i, j=1,2) .
$$

Let

$$
\begin{aligned}
& H_{1}=\left\{\operatorname{Ad}\left(\begin{array}{cccc}
A & 0 & B & 0 \\
0 & 1 & 0 & 0 \\
-\bar{B} & 0 & \bar{A} & 0 \\
0 & 0 & 0 & 1
\end{array}\right) \in K_{\mathrm{R}}\right\}, \\
& H_{2}=\left\{\operatorname{Ad}\left(\begin{array}{cccc}
I_{p} & 0 & 0 & 0 \\
0 & \alpha & 0 & \beta \\
0 & 0 & I_{p} & 0 \\
0 & -\bar{\beta} & 0 & \bar{\alpha}
\end{array}\right) \in K_{\mathbf{R}}\right\} .
\end{aligned}
$$

Then $H_{1}$ and $H_{2}$ are subgroups of $K_{\mathrm{R}}$, and for any $g \in K_{\mathrm{R}}$ there are unique $h_{j} \in H_{j}$ $(j=1,2)$ such that $g=h_{1} h_{2}$. If $g_{j} \in H_{j}(j=1,2)$, we have $g_{1} g_{2}=g_{2} g_{1}$. From the proof of Lemma $2.1 \Sigma=H_{1} E_{0}$. We have from (2.3)

$$
\begin{array}{r}
H_{1} \tilde{E}_{r}=\left\{\Phi^{-1}\left(\begin{array}{c}
x \\
z \\
y \\
w
\end{array}\right) ;\left(\begin{array}{l}
x \\
z
\end{array}\right) \cdot\left(\begin{array}{l}
y \\
w
\end{array}\right)=\left(\begin{array}{c}
x \\
z
\end{array}\right) \cdot\left(\begin{array}{c}
w \\
-y
\end{array}\right)=0,\right. \\
\left.x \cdot \bar{x}+z \cdot \bar{z}=r^{2}, y \cdot \bar{y}+w \cdot \bar{w}=1-r^{2}\right\} .
\end{array}
$$

We denote by $d \mu_{j}$ the Haar measures on $H_{j}$ such that $\int_{H_{j}} 1 d \mu_{j}=1(j=1,2)$. If $f$ and $h$ are continuous functions on $\Sigma$ and $N(r)$ respectively, then we have

$$
\int_{H_{1}} f\left(g E_{0}\right) d \mu_{1}(g)=\int_{\Sigma} f(X) d \mu_{\Sigma}(X)
$$

and

$$
\int_{N(r)} h(Z) d \mu_{r}(Z)=\int_{H_{2}}\left(\int_{H_{1}} h\left(g_{1} g_{2} \tilde{E}_{r}\right) d \mu_{1}\left(g_{1}\right)\right) d \mu_{2}\left(g_{2}\right) .
$$

We define the function $\rho$ on $[0,1]$ by 


$$
\rho(r)=2^{4 p-3} \frac{\Gamma\left(2 p-\frac{1}{2}\right)}{\pi^{1 / 2} \Gamma(2 p-2)} r^{4 p-5}\left(1-r^{2}\right)^{2 p-3}\left(2 r^{2}-1\right)^{2} .
$$

Then our main theorem in this section is stated as follows:

THEOREM 2.2. (i) For $f \in \mathscr{H}_{m}$ and $X \in \Sigma$ we have

$$
\operatorname{dim} \mathscr{H}_{n} \int_{\Sigma} f(Y) Q_{n, p}(Y, X) d \mu_{\Sigma}(Y)=\delta_{m, n} f(X) .
$$

(ii) For any $X \in \mathfrak{p}$ and $Y \in \Sigma$ we have

$$
\int_{0}^{1} \rho(r)\left(\int_{N(r)}\langle Z, Y\rangle^{m}\langle\overline{Z, X}\rangle^{n} d \mu_{r}(Z)\right) d r=\frac{n ! \Gamma(2 p)}{\Gamma(n+2 p)} \delta_{m, n} Q_{n, p}(X, Y) .
$$

(iii) For any $f \in \mathscr{H}_{m}$ and any $X \in \mathfrak{p}$ we have

$$
\int_{0}^{1} \rho(r)\left(\int_{N(r)} f(Z)\langle\overline{Z, X}\rangle^{n} d \mu_{r}(Z)\right) d r=\left(\operatorname{dim} \mathscr{H}_{n}\right)^{-1} \delta_{m, n} f(X) .
$$

(iv) For any $f \in \mathscr{H}_{m}$ and $g \in \mathscr{H}_{n}$ we have

$$
\int_{\Sigma} f(X) \overline{g(X)} d \mu_{\Sigma}(X)=\frac{n ! \Gamma(2 p) \operatorname{dim} \mathscr{H}_{n}}{\Gamma(n+2 p)} \int_{0}^{1} \rho(r)\left(\int_{N(r)} f(X) \overline{g(X)} d \mu_{r}(X)\right) d r .
$$

To prove Theorem 2.2, we need some lemmas.

LEMMA 2.3 ([2], [5], [7], [15]). (i) For $\theta \in \mathbf{R}$ we have $P_{n, 4 p}(\cos \theta)=\frac{2^{4 p-2} \Gamma\left(2 p-\frac{1}{2}\right)}{\pi^{1 / 2} \Gamma(2 p-1)} \int_{0}^{1}\left\{\cos \theta+\left(2 r^{2}-1\right) i \sin \theta\right\}^{n} r^{4 p-3}\left(1-r^{2}\right)^{2 p-2} d r$

(ii) Let $\alpha, \beta \in \mathrm{C}^{4 p}, \alpha \cdot \alpha=\beta \cdot \beta=0$. Then we have

$$
\int_{S^{4 p-1}}(s \cdot \alpha)^{m}(\overline{s \cdot \beta})^{n} d s=\frac{2^{-n} n ! \Gamma(2 p)}{\Gamma(n+2 p)} \delta_{m, n}(\alpha \cdot \bar{\beta})^{n} .
$$

(iii) If $X$ and $Y$ belong to $\mathfrak{N}$, then we get

$$
\int_{\Sigma}\langle X, Z\rangle^{m}\langle\overline{Y, Z}\rangle^{n} d \mu_{\Sigma}(Z)=\frac{n ! \Gamma(2 p)}{\Gamma(n+2 p)} \delta_{m, n}\langle X, Y\rangle^{n} .
$$

Proof. (i) It is well known that the following formula holds for $0 \leq t \leq 1$ :

$$
P_{n, 4 p}(t)=\frac{\Gamma\left(2 p-\frac{1}{2}\right)}{\pi^{1 / 2} \Gamma(2 p-1)} \int_{-1}^{1}\left\{t \pm i\left(1-t^{2}\right)^{1 / 2} x\right\}^{n}\left(1-x^{2}\right)^{2 p-2} d x
$$


(see [5], [7]). From this formula we get (2.10) by putting $r=\sqrt{(x+1) / 2}$.

(ii) Suppose $\alpha \cdot \alpha=\beta \cdot \beta=0$. Then we have $(z \cdot \alpha)^{m} \in H_{m}\left(\mathbf{C}^{4 p}\right)$ and $(z \cdot \bar{\beta})^{n} \in H_{n}\left(\mathbf{C}^{4 p}\right)$. Hence, (2.11) follows from (1.2) and (1.3).

(iii) We put $X=\Phi^{-1}\left(\begin{array}{c}c_{1} \\ c_{2} \\ d_{1} \\ d_{2}\end{array}\right), \quad Y=\Phi^{-1}\left(\begin{array}{c}\mu_{1} \\ \mu_{2} \\ \eta_{1} \\ \eta_{2}\end{array}\right) \in \mathfrak{N}$ and $Z=\Phi^{-1}\left(\begin{array}{c}x \\ z \\ \bar{x} \\ \bar{z}\end{array}\right) \in \Sigma(x=$ $\left.x_{1}+i x_{2}, z=z_{1}+i z_{2}, x_{1}, x_{2}, z_{1}, z_{2} \in \mathbf{R}^{p}\right)$. Then we have by (2.11)

$$
\begin{aligned}
& \int_{\Sigma}\langle X, Z\rangle^{m}\langle\overline{Y, Z}\rangle^{n} d \mu_{\Sigma}(Z) \\
& =\int_{S^{4 p-1}}\left\{\left(\begin{array}{c}
c_{1}+d_{1} \\
c_{2}+d_{2} \\
i\left(d_{1}-c_{1}\right) \\
i\left(d_{2}-c_{2}\right)
\end{array}\right) \cdot\left(\begin{array}{c}
x_{1} \\
z_{1} \\
x_{2} \\
z_{2}
\end{array}\right)\right\}\left\{\left(\begin{array}{c}
\overline{\mu_{1}+\eta_{1}} \\
\mu_{2}+\eta_{2} \\
i\left(\eta_{1}-\mu_{1}\right) \\
i\left(\eta_{2}-\mu_{2}\right)
\end{array}\right) \cdot\left(\begin{array}{c}
x_{1} \\
z_{1} \\
x_{2} \\
z_{2}
\end{array}\right)\right\}^{n} d \mu_{S} \\
& =\frac{2^{-n} n ! \Gamma(p)}{\Gamma(n+p)} \delta_{m, n}\left(\sum_{j=1}^{2}\left\{\left(c_{j}+d_{j}\right) \cdot\left(\bar{\mu}_{j}+\bar{\eta}_{j}\right)+\left(c_{j}-d_{j}\right) \cdot\left(\bar{\mu}_{j}-\bar{\eta}_{j}\right)\right\}\right)^{n} \\
& =\frac{n ! \Gamma(2 p)}{\Gamma(n+2 p)} \delta_{m, n}\langle X, Y\rangle^{n}
\end{aligned}
$$

because

$$
\begin{aligned}
& \sum_{j=1}^{2}\left\{\left(c_{j}+d_{j}\right) \cdot\left(c_{j}+d_{j}\right)-\left(d_{j}-c_{j}\right) \cdot\left(d_{j}-c_{j}\right)\right\} \\
& =\sum_{j=1}^{2}\left\{\left(\mu_{j}+\eta_{j}\right) \cdot\left(\mu_{j}+\eta_{j}\right)-\left(\eta_{j}-\mu_{j}\right) \cdot\left(\eta_{j}-\mu_{j}\right)\right\}=0 .
\end{aligned}
$$

Q.E.D.

LEMMA 2.4. We put

$$
G_{0}=\left\{\left(\begin{array}{cccc}
\alpha & 0 & -\beta & 0 \\
0 & \alpha & 0 & \beta \\
\bar{\beta} & 0 & \bar{\alpha} & 0 \\
0 & -\bar{\beta} & 0 & \bar{\alpha}
\end{array}\right) \in U(4) ; \alpha \bar{\alpha}+\beta \bar{\beta}=1\right\}
$$

and

$$
\mathfrak{p}_{0}=\left\{\left(\begin{array}{cccc}
0 & x_{1} & 0 & w_{1} \\
y_{1} & 0 & w_{1} & 0 \\
0 & z_{1} & 0 & -y_{1} \\
z_{1} & 0 & -x_{1} & 0
\end{array}\right) \in M(4, \mathrm{C})\right\}
$$

We denote by $J^{\prime}\left(\mathfrak{p}_{0}\right)$ the ring of $\operatorname{Ad} G_{0}$-invariant polynomials with complex coefficients on 
$\mathfrak{p}_{0}$. If $f \in J^{\prime}\left(\mathfrak{p}_{0}\right)$, then we have for $X=\left(\begin{array}{cccc}0 & x_{1} & 0 & w_{1} \\ y_{1} & 0 & w_{1} & 0 \\ 0 & z_{1} & 0 & -y_{1} \\ z_{1} & 0 & -x_{1} & 0\end{array}\right) \in \mathfrak{p}_{0}$

$$
f(X)=\varphi\left(x_{1}+y_{1}, x_{1} y_{1}+z_{1} w_{1}\right),
$$

where $\varphi$ is some polynomial on $\mathbf{C}^{2}$.

Proof. Let $g_{0}$ be the Lie algebra of the group $G_{0}$. Then we have

$$
\mathfrak{g}_{0}=\left\{\left(\begin{array}{cccc}
\alpha & 0 & -\beta & 0 \\
0 & \alpha & 0 & \beta \\
\bar{\beta} & 0 & \bar{\alpha} & 0 \\
0 & -\bar{\beta} & 0 & \bar{\alpha}
\end{array}\right) ; \alpha, \beta \in \mathbf{C}, \alpha+\bar{\alpha}=0\right\} .
$$

Let $S^{\prime}\left(\mathfrak{p}_{0}\right)$ be the space of polynomials with complex coefficients on $\mathfrak{p}_{0}$ and let $S_{m}^{\prime}\left(\mathfrak{p}_{0}\right)$ be the space of homogeneous polynomials on $\mathfrak{p}_{0}$ of degree $m$. Remark that $S_{1}^{\prime}\left(\mathfrak{p}_{0}\right)$ is isomorphic to $\mathfrak{p}_{0}^{*}$. Since $G_{0}$ is connected, $f \in S^{\prime}\left(\mathfrak{p}_{0}\right)$ belongs to $J^{\prime}\left(\mathfrak{p}_{0}\right)$ if and only if $-(\operatorname{ad} Y)^{*} f=0$ for any $Y \in \mathfrak{g}_{0}$, where $(\operatorname{ad} Y)^{*}: S^{\prime}\left(\mathfrak{p}_{0}\right) \rightarrow S^{\prime}\left(\mathfrak{p}_{0}\right)$ is the map naturally induced by the dual of ad $Y: \mathfrak{p}_{0} \rightarrow \mathfrak{p}_{0}$. We put

$$
\begin{aligned}
& Y_{1}=\left(\begin{array}{cccc}
i & 0 & 0 & 0 \\
0 & i & 0 & 0 \\
0 & 0 & -i & 0 \\
0 & 0 & 0 & -i
\end{array}\right), \quad Y_{2}=\left(\begin{array}{cccc}
0 & 0 & -1 & 0 \\
0 & 0 & 0 & 1 \\
1 & 0 & 0 & 0 \\
0 & -1 & 0 & 0
\end{array}\right), \quad Y_{3}=\left(\begin{array}{cccc}
0 & 0 & -i & 0 \\
0 & 0 & 0 & i \\
-i & 0 & 0 & 0 \\
0 & i & 0 & 0
\end{array}\right), \\
& X_{1}=\left(\begin{array}{cccc}
0 & 1 & 0 & 0 \\
0 & 0 & 0 & 0 \\
0 & 0 & 0 & 0 \\
0 & 0 & -1 & 0
\end{array}\right), \quad X_{2}=\left(\begin{array}{cccc}
0 & 0 & 0 & 0 \\
1 & 0 & 0 & 0 \\
0 & 0 & 0 & -1 \\
0 & 0 & 0 & 0
\end{array}\right), \quad X_{3}=\left(\begin{array}{llll}
0 & 0 & 0 & 0 \\
0 & 0 & 0 & 0 \\
0 & 1 & 0 & 0 \\
1 & 0 & 0 & 0
\end{array}\right), \\
& X_{4}=\left(\begin{array}{llll}
0 & 0 & 0 & 1 \\
0 & 0 & 1 & 0 \\
0 & 0 & 0 & 0 \\
0 & 0 & 0 & 0
\end{array}\right)
\end{aligned}
$$

$\left\{Y_{j} ; 1 \leq j \leq 3\right\}$ is a basis of $\mathfrak{g}_{0} .\left\{X_{j} ; 1 \leq j \leq 4\right\}$ is a basis of $\mathfrak{p}_{0}$ and $\left\{X_{j}^{*} ; 1 \leq j \leq 4\right\}$ denotes its dual basis of $\mathfrak{p}_{0}^{*}$. Clearly, we have $X_{1}^{*}=x_{1}, X_{2}^{*}=y_{1}, X_{3}^{*}=z_{1}, X_{4}^{*}=w_{1}$. By using the bracket table 


\begin{tabular}{c|c|c|c|c}
\hline & $X_{1}$ & $X_{2}$ & $X_{3}$ & $X_{4}$ \\
\hline$Y_{1}$ & 0 & 0 & $-2 i X_{3}$ & $2 i X_{4}$ \\
\hline$Y_{2}$ & $X_{3}-X_{4}$ & $-X_{3}+X_{4}$ & $-X_{1}+X_{2}$ & $X_{1}-X_{2}$ \\
\hline$Y_{3}$ & $-i X_{3}-i X_{4}$ & $i X_{3}+i X_{4}$ & $-i X_{1}+i X_{2}$ & $-i X_{1}+i X_{2}$ \\
\hline
\end{tabular}

we have $-\left(\operatorname{ad} Y_{1}\right)^{*} X_{1}^{*}=-\left(\operatorname{ad} Y_{1}\right)^{*} X_{2}^{*}=0,-\left(\operatorname{ad} Y_{1}\right) * X_{3}^{*}=2 i X_{3}^{*}$ and $-\left(\operatorname{ad} Y_{1}\right)^{*} X_{4}^{*}=$ $-2 i X_{4}^{*}$. In particular, we see that the space of $-\left(\operatorname{ad} Y_{1}\right)^{*}$-invariant polynomials is generated by $\left\{X_{1}^{*}, X_{2}^{*}, X_{3}^{*} X_{4}^{*}\right\}$.

Next, from the above table, we have

$$
\begin{aligned}
& -\left(\operatorname{ad} Y_{2}\right) * X_{1}^{*}=X_{3}^{*}-X_{4}^{*}, \\
& -\left(\operatorname{ad} Y_{2}\right) * X_{2}^{*}=-X_{3}^{*}+X_{4}^{*}, \\
& -\left(\operatorname{ad} Y_{2}\right) * X_{3}^{*}=-X_{1}^{*}+X_{2}^{*}, \\
& -\left(\operatorname{ad} Y_{2}\right) * X_{4}^{*}=X_{1}^{*}-X_{2}^{*} .
\end{aligned}
$$

Hence, the eigenvalues of the linear transform $-\left(\operatorname{ad} Y_{2}\right)^{*}$ on $S_{1}^{\prime}\left(\mathfrak{p}_{0}\right)$ are $0,2 i$, and $-2 i$ and we have $V(0)=\left\langle X_{1}^{*}+X_{2}^{*}, X_{3}^{*}+X_{4}^{*}\right\rangle, V(2 i)=\left\langle i X_{1}^{*}-i X_{2}^{*}+X_{3}^{*}-X_{4}^{*}\right\rangle$, and $V(-2 i)=$ $\left\langle i X_{1}^{*}-i X_{2}^{*}-X_{3}^{*}+X_{4}^{*}\right\rangle$, where $V(\lambda)$ is the eigenspace corresponding to the eigenvalue $\lambda$. Therefore, the space of $-\left(\operatorname{ad} Y_{2}\right)^{*}$-invariant polynomials is generated by $\left\{X_{1}^{*}+\right.$ $\left.X_{2}^{*}, X_{3}^{*}+X_{4}^{*}, X_{1}^{*} X_{2}^{*}+X_{3}^{*} X_{4}^{*}\right\}$. From these facts, we can express $f \in J^{\prime}\left(\mathfrak{p}_{0}\right)$ in the form

$$
\begin{aligned}
f & =\sum_{k, l, m} A_{k, l, m}\left(X_{1}^{*}\right)^{k}\left(X_{2}^{*}\right)^{l}\left(X_{3}^{*} X_{4}^{*}\right)^{m} \\
& =\sum_{r \geq 0}\left(X_{3}^{*}+X_{4}^{*}\right)^{r} \varphi_{r}\left(X_{1}^{*}+X_{2}^{*}, X_{1}^{*} X_{2}^{*}+X_{3}^{*} X_{4}^{*}\right),
\end{aligned}
$$

where $\varphi_{r}$ is a polynomial of $X_{1}^{*}+X_{2}^{*}$ and $X_{1}^{*} X_{2}^{*}+X_{3}^{*} X_{4}^{*}$, and $A_{k, l, m} \in \mathbf{C}$. Then, since the term corresponding to $r=0$ is expressed as a polynomial of $X_{1}^{*}, X_{2}^{*}, X_{3}^{*} X_{4}^{*}$, we have

$$
\begin{gathered}
\sum_{r \geq 1}\left(X_{3}^{*}+X_{4}^{*}\right)^{r} \varphi_{r}\left(X_{1}^{*}+X_{2}^{*}, X_{1}^{*} X_{2}^{*}+X_{3}^{*} X_{4}^{*}\right) \\
=\sum_{k, l, m} B_{k, l, m}\left(X_{1}^{*}\right)^{k}\left(X_{2}^{*}\right)^{l}\left(X_{3}^{*} X_{4}^{*}\right)^{m},
\end{gathered}
$$

where $B_{k, l, m} \in \mathbf{C}$. By putting $X_{3}^{*}=-X_{4}^{*}$, we have from this equality

$$
\sum_{k, l, m} B_{k, l, m}\left(X_{1}^{*}\right)^{k}\left(X_{2}^{*}\right)^{l}\left(-X_{3}^{*}\right)^{2 m}=0
$$

Hence, $B_{k, l, m}=0$ and we get $f=\varphi_{0}\left(X_{1}^{*}+X_{2}^{*}, X_{1}^{*} X_{2}^{*}+X_{3}^{*} X_{4}^{*}\right)$. The invariance by 
$-\left(\operatorname{ad} Y_{3}\right)^{*}$ follows immediately from that of $-\left(\operatorname{ad} Y_{1}\right)^{*}$ and $-\left(\operatorname{ad} Y_{2}\right)^{*}$.

Q.E.D.

LEMMA 2.5. Let $L_{n}$ be a homogeneous polynomial on $\mathfrak{p}$ of degree $n$ with complex coefficients. If $L_{n}(k X)=L_{n}(X)$ for any $k \in K_{0}$, then $L_{n}(X)$ is expressed as follows:

$$
L_{n}(X)=\sum_{(l, r) \in \Lambda} C_{l, r}\left(x_{1}+y_{1}\right)^{l}\left(x_{1} y_{1}+z_{1} w_{1}\right)^{r}\left(x^{\prime} \cdot y^{\prime}+z^{\prime} \cdot w^{\prime}\right)^{(n-l-2 r) / 2},
$$

where $\Lambda=\left\{(l, r) \in \mathbf{Z}_{+}^{2} ; n \equiv l(\bmod 2), l+2 r \leq n\right\}, C_{l, r} \in \mathbf{C}, X=\Phi^{-1}\left(\begin{array}{c}x \\ z \\ y \\ w\end{array}\right) \in \mathfrak{p}, x=\left(\begin{array}{c}x_{1} \\ x^{\prime}\end{array}\right)$, $y=\left(\begin{array}{c}y_{1} \\ y^{\prime}\end{array}\right), z=\left(\begin{array}{c}z_{1} \\ z^{\prime}\end{array}\right), w=\left(\begin{array}{c}w_{1} \\ w^{\prime}\end{array}\right), x_{1}, y_{1}, z_{1}, w_{1} \in \mathbf{C}$ and $x^{\prime}, y^{\prime}, z^{\prime}, w^{\prime} \in \mathbf{C}^{p-1}$.

Proof. Since $L_{n}$ is a homogeneous polynomial of degree $n, L_{n}$ can be expressed as follows:

$$
L_{n}(X)=\sum_{\substack{0 \leq q, r, s, t \leq n \\ 0 \leq q+r+s+t \leq n}} x_{1}^{q} y_{1}^{r} z_{1}^{s} w_{1}^{t} A_{q, r, s, t}\left(t^{t}\left(x^{\prime} z^{\prime} y^{\prime} w^{\prime}\right)\right)
$$

where $A_{q, r, s, t}$ is a homogeneous polynomial of $\left(\begin{array}{c}x^{\prime} \\ z^{\prime} \\ y^{\prime} \\ w^{\prime}\end{array}\right)$ of degree $n-(q+r+s+t)$.

For any $k=\operatorname{Ad}\left(\begin{array}{cccccc}\alpha & 0 & 0 & -\beta & 0 & 0 \\ 0 & A^{\prime} & 0 & 0 & B^{\prime} & 0 \\ 0 & 0 & \alpha & 0 & 0 & \beta \\ \bar{\beta} & 0 & 0 & \bar{\alpha} & 0 & 0 \\ 0 & -\bar{B}^{\prime} & 0 & 0 & \bar{A}^{\prime} & 0 \\ 0 & 0 & -\bar{\beta} & 0 & 0 & \bar{\alpha}\end{array}\right) \in K_{0}$ we put $\left(\begin{array}{c}\chi \\ \zeta \\ \xi \\ v\end{array}\right)=\Phi(k X), \chi=\left(\begin{array}{c}\chi_{1} \\ \chi^{\prime}\end{array}\right), \zeta=\left(\begin{array}{c}\zeta_{1} \\ \zeta^{\prime}\end{array}\right)$, $\xi=\left(\begin{array}{l}\xi_{1} \\ \xi^{\prime}\end{array}\right), v=\left(\begin{array}{l}v_{1} \\ v^{\prime}\end{array}\right)$. Then we have from (2.2)

$$
\left(\begin{array}{c}
\chi^{\prime} \\
\zeta^{\prime} \\
\xi^{\prime} \\
v^{\prime}
\end{array}\right)=\left(\begin{array}{cc}
\bar{\alpha} A_{1}^{\prime} & -\bar{\beta} A_{2}^{\prime} \\
-\beta \bar{A}_{2}^{\prime} & \alpha \bar{A}_{1}^{\prime}
\end{array}\right)\left(\begin{array}{c}
x^{\prime} \\
z^{\prime} \\
y^{\prime} \\
w^{\prime}
\end{array}\right),
$$

where we put $A_{1}^{\prime}=\left(\begin{array}{cc}A^{\prime} & B^{\prime} \\ -\bar{B}^{\prime} & \bar{A}^{\prime}\end{array}\right), A_{2}^{\prime}=\left(\begin{array}{cc}B^{\prime} & -A^{\prime} \\ \bar{A}^{\prime} & \bar{B}^{\prime}\end{array}\right)$. Furthermore $g=\operatorname{Ad}\left(\begin{array}{cccc}\alpha & 0 & -\beta & 0 \\ 0 & \alpha & 0 & \beta \\ \bar{\beta} & 0 & \bar{\alpha} & 0 \\ 0 & -\bar{\beta} & 0 & \bar{\alpha}\end{array}\right)$ belongs to $\operatorname{Ad} G_{0}$. Remark that $\left(\begin{array}{cc}\bar{\alpha} A_{1}^{\prime} & -\bar{\beta} A_{2}^{\prime} \\ -\beta \bar{A}_{2}^{\prime} & \alpha \bar{A}_{1}^{\prime}\end{array}\right)$ belongs to $U(4 p-4)$. The action of $g \in \operatorname{Ad} G_{0}$ on the space $\mathfrak{p}_{0}$ can be expressed as follows: 


$$
\left(\begin{array}{cccc}
0 & \chi_{1} & 0 & v_{1} \\
\xi_{1} & 0 & v_{1} & 0 \\
0 & \zeta_{1} & 0 & -\xi_{1} \\
\zeta_{1} & 0 & -\chi_{1} & 0
\end{array}\right)=g\left(\left(\begin{array}{cccc}
0 & x_{1} & 0 & w_{1} \\
y_{1} & 0 & w_{1} & 0 \\
0 & z_{1} & 0 & -y_{1} \\
z_{1} & 0 & -x_{1} & 0
\end{array}\right)\right)
$$

We put

$$
\begin{gathered}
H_{1}^{\prime}=\left\{\left(\begin{array}{cc}
A_{1}^{\prime} & 0 \\
0 & \bar{A}_{1}^{\prime}
\end{array}\right) \in U(4 p-4) ; A_{1}^{\prime}=\left(\begin{array}{cc}
A^{\prime} & B^{\prime} \\
-\bar{B}^{\prime} & \bar{A}^{\prime}
\end{array}\right) \in U(2 p-2),\right. \\
\left.A^{\prime}, B^{\prime} \in M(p-1, \mathbf{C})\right\}
\end{gathered}
$$

When $\alpha=1$ and $\beta=0$, from (2.14) and (2.15) it is valid

$$
L_{n}(X)=\sum_{\substack{0 \leq q, r, s, t \leq n \\ 0 \leq q+r+s+t \leq n}} x_{1}^{q} y_{1}^{r} z_{1}^{s} w_{1}^{t} A_{q, r, s, t}\left(h^{t}\left(x^{\prime} z^{\prime} y^{\prime} w^{\prime}\right)\right)
$$

for any $h \in H_{1}^{\prime}$ because $L_{n}(X)$ is $K_{0}$-invariant. This implies

$$
\left.A_{q, r, s, t}\left(t^{\prime} z^{\prime} y^{\prime} w^{\prime}\right)\right)=A_{q, r, s, t}\left(h^{t}\left(x^{\prime} z^{\prime} y^{\prime} w^{\prime}\right)\right)
$$

for any $h \in H_{1}^{\prime}$. We put $\Sigma^{\prime}=\left\{\left(\begin{array}{c}x^{\prime} \\ z^{\prime} \\ y^{\prime} \\ w^{\prime}\end{array}\right) \in \mathbf{C}^{4 p-4} ;\left(\begin{array}{c}y^{\prime} \\ w^{\prime}\end{array}\right)=\left(\begin{array}{c}x^{\prime} \\ z^{\prime}\end{array}\right),\left(\begin{array}{c}x^{\prime} \\ z^{\prime}\end{array}\right) \cdot\left(\begin{array}{c}x^{\prime} \\ z^{\prime}\end{array}\right)=1\right\}$. We can prove that $H_{1}^{\prime}$ acts on $\Sigma^{\prime}$ transitively similarly to the proof of Lemma 2.1 . Hence $A_{q, r, s, t}$ is constant on $\Sigma^{\prime}$ by (2.16). For $X=\left(\begin{array}{c}x^{\prime} \\ z^{\prime} \\ y^{\prime} \\ w^{\prime}\end{array}\right) \in \mathbf{C}^{4 p-4}$ we define the mapping $\Psi_{1}: \mathrm{C}^{4 p-4} \rightarrow \mathrm{C}^{4 p-4}$ by

$$
\Psi_{1}(X)=\frac{1}{2}\left(\begin{array}{c}
x^{\prime}+y^{\prime} \\
z^{\prime}+w^{\prime} \\
-i\left(x^{\prime}-y^{\prime}\right) \\
-i\left(z^{\prime}-w^{\prime}\right)
\end{array}\right)
$$

Then it is clear that $\Psi_{1}$ is the bijection from $\Sigma^{\prime}$ onto $S^{4(p-1)-1}$. Hence, $A_{q, r, s, t} \circ \Psi_{1}^{-1}$ is constant on $S^{4(p-1)-1}$. Since $A_{q, r, s, t} \circ \Psi_{1}^{-1}$ is a homogeneous polynomial on $\mathrm{C}^{4 p-4}$, we have for ${ }^{t}\left(\lambda_{1} \lambda_{2} \lambda_{3} \lambda_{4}\right) \in \mathbf{R}^{4 p-4}$

$$
A_{q, r, s, t} \circ \Psi_{1}^{-1}\left({ }^{t}\left(\lambda_{1} \lambda_{2} \lambda_{3} \lambda_{4}\right)\right)=C_{q, r, s, t}\left(\sum_{j=1}^{4} \lambda_{j}^{2}\right)^{(n-q-r-s-t) / 2},
$$

where $C_{q, r, s, t} \in \mathbf{C}$, and $C_{q, r, s, t}=0$ when $n-q-r-s-t$ is odd. Since $A_{q, r, s, t} \circ \Psi_{1}^{-1}$ is holomorphic on $\mathbf{C}^{4 p-4},(2.17)$ is valid for any ${ }^{t}\left(\lambda_{1} \lambda_{2} \lambda_{3} \lambda_{4}\right) \in \mathbf{C}^{4 p-4}$. Therefore we get 


$$
A_{q, r, s, t}\left(t^{t}\left(x^{\prime} z^{\prime} y^{\prime} w^{\prime}\right)\right)=C_{q, r, s, t}\left(x^{\prime} \cdot y^{\prime}+z^{\prime} \cdot w^{\prime}\right)^{(n-q-r-s-t) / 2} .
$$

From this equation we can write

$$
\begin{aligned}
& L_{n}(X)=\sum_{\substack{0 \leq q, r, s, t \leq n \\
0 \leq q+r+s+t \leq n}} x_{1}^{q} y_{1}^{r} z_{1}^{s} w_{1}^{t} C_{q, r, s, t}\left(x^{\prime} \cdot y^{\prime}+z^{\prime} \cdot w^{\prime}\right)^{(n-q-r-s-t) / 2} \\
& =\sum_{k=0}^{[n / 2]}\left\{\sum_{q+r+s+t=n-2 k} C_{q, r, s, t} x_{1}^{q} y_{1}^{r} z_{1}^{s} w_{1}^{t}\right\}\left(x^{\prime} \cdot y^{\prime}+z^{\prime} \cdot w^{\prime}\right)^{k} \\
& =\sum_{k=0}^{[n / 2]} f_{n-2 k}\left({ }^{t}\left(x_{1} z_{1} y_{1} w_{1}\right)\right)\left(x^{\prime} \cdot y^{\prime}+z^{\prime} \cdot w^{\prime}\right)^{k} \text {, }
\end{aligned}
$$

where $f_{n-2 k}$ is a homogeneous polynomial of degree $n-2 k$. Next, we consider the action of $k \in K_{0}$ on $L_{n}(X)$, not assuming $\alpha=1, \beta=0$. (2.18) and (2.14) imply that

$$
\begin{aligned}
L_{n}(X) & =\sum_{k=0}^{[n / 2]} f_{n-2 k}\left({ }^{t}\left(\chi_{1} \zeta_{1} \xi_{1} v_{1}\right)\right)\left(\chi^{\prime} \cdot \xi^{\prime}+\zeta^{\prime} \cdot v^{\prime}\right)^{k} \\
& =\sum_{k=0}^{[n / 2]} f_{n-2 k}\left({ }^{t}\left(\chi_{1} \zeta_{1} \xi_{1} v_{1}\right)\right)\left(x^{\prime} \cdot y^{\prime}+z^{\prime} \cdot w^{\prime}\right)^{k}
\end{aligned}
$$

because $x^{\prime} \cdot y^{\prime}+z^{\prime} \cdot w^{\prime}$ is $K_{0}$-invariant. Hence, we have

$$
f_{n-2 k}\left({ }^{t}\left(\chi_{1} \zeta_{1} \xi_{1} v_{1}\right)\right)=f_{n-2 k}\left({ }^{t}\left(x_{1} z_{1} y_{1} w_{1}\right)\right) \text {, }
$$

which implies that $f_{n-2 k}$ is $G_{0}$-invariant on account of (2.15). Then by Lemma 2.4 we obtain

$$
f_{n-2 k}\left({ }^{t}\left(x_{1} z_{1} y_{1} w_{1}\right)\right)=\sum_{l+2 r=n-2 k} C_{l, r}\left(x_{1}+y_{1}\right)^{l}\left(x_{1} y_{1}+z_{1} w_{1}\right)^{r} .
$$

From (2.18) and (2.19) we get (2.13).

Q.E.D.

LEMMA 2.6. For any $X \in \mathfrak{p}$ we have

$$
\int_{0}^{1} \rho(r)\left(\int_{N(r)}\left\langle Y, E_{0}\right\rangle^{m}\langle\overline{Y, X}\rangle^{n} d \mu_{r}(Y)\right) d r=\frac{n ! \Gamma(2 p)}{\Gamma(n+2 p)} \delta_{m, n} Q_{n, p}\left(X, E_{0}\right) .
$$

Proof. For any $X \in \mathfrak{p}$ we put

$$
F(X)=\int_{0}^{1} \rho(r)\left(\int_{N(r)}\left\langle Y, E_{0}\right\rangle^{m}\langle\overline{Y, X}\rangle^{n} d \mu_{r}(Y)\right) d r .
$$

Then $F(X)$ belongs to $\mathscr{H}_{n}$ because $N(r) \subset \mathfrak{N}$. Furthermore, $F(X)$ is $K_{0}$-invariant because the inner product $\langle$,$\rangle and d \mu_{r}$ are $K_{\mathrm{R}}$-invariant. Hence, by Lemma $2.5, F(X)$ can be expressed as follows:

$$
F(X)=\sum_{(l, r) \in \Lambda} C_{l, r}\left(x_{1}+y_{1}\right)^{l}\left(x_{1} y_{1}+z_{1} w_{1}\right)^{r}\left(x^{\prime} \cdot y^{\prime}+z^{\prime} \cdot w^{\prime}\right)^{(n-l-2 r) / 2} .
$$


We put $X_{\theta}=\Phi^{-1}\left(\begin{array}{c}e^{i \theta} e_{1} \\ 0 \\ e^{-i \theta} e_{1} \\ 0\end{array}\right) \in \Sigma, h_{\theta}^{-1}=\operatorname{Ad}\left(\begin{array}{cccc}I_{p} & 0 & 0 & 0 \\ 0 & e^{-i \theta} & 0 & 0 \\ 0 & 0 & I_{p} & 0 \\ 0 & 0 & 0 & e^{i \theta}\end{array}\right) \in H_{2}$. Then we have $X_{\theta}=$ $h_{\theta}^{-1} E_{0}$ and $g h_{\theta}=h_{\theta} g$ for any $g \in H_{1}$. We put

$$
G_{r}\left(X_{\theta}\right)=\int_{N(r)}\left\langle Y, E_{0}\right\rangle^{m}\left\langle\overline{Y, X_{\theta}}\right\rangle^{n} d \mu_{r}(Y) .
$$

Then it is valid that for any $g \in H_{1}$

$$
\begin{aligned}
G_{r}\left(X_{\theta}\right) & =\int_{N(r)}\left\langle Y, E_{0}\right\rangle^{m}\left\langle\overline{Y, h_{\theta}^{-1} E_{0}}\right\rangle^{n} d \mu_{r}(Y) \\
& =\int_{N(r)}\left\langle Y, E_{0}\right\rangle^{m}\left\langle\overline{\left\langle h_{\theta} Y, E_{0}\right.}\right\rangle^{n} d \mu_{r}(Y) \\
& =\int_{N(r)}\left\langle g^{-1} Y, E_{0}\right\rangle^{m}\left\langle\overline{h_{\theta} g^{-1} Y, E_{0}}\right\rangle^{n} d \mu_{r}(Y) \\
& =\int_{N(r)}\left\langle g^{-1} Y, E_{0}\right\rangle^{m}\left\langle\overline{\left\langle g^{-1} h_{\theta} Y, E_{0}\right.}\right\rangle^{n} d \mu_{r}(Y) \\
& =\int_{N(r)}\left\langle Y, g E_{0}\right\rangle^{m}\left\langle\overline{\left\langle h_{\theta} Y, g E_{0}\right.}\right\rangle^{n} d \mu_{r}(Y) .
\end{aligned}
$$

From this equality we have

$$
\begin{aligned}
G_{r}\left(X_{\theta}\right) & =\int_{H_{1}} G_{r}\left(X_{\theta}\right) d \mu_{1}(g) \\
& =\int_{H_{1}}\left(\int_{N(r)}\left\langle Y, g E_{0}\right\rangle^{m}\left\langle\overline{h_{\theta} Y, g E_{0}}\right\rangle^{n} d \mu_{r}(Y)\right) d \mu_{1}(g) \\
& =\int_{N(r)}\left(\int_{H_{1}}\left\langle Y, g E_{0}\right\rangle^{m}\left\langle\overline{h_{\theta} Y, g E_{0}}\right\rangle^{n} d \mu_{1}(g)\right) d \mu_{r}(Y) .
\end{aligned}
$$

Since $H_{1} E_{0}=\Sigma$, and $Y$ and $h_{\theta} Y$ belong to $N(r) \subset \mathfrak{N}$, we have from (2.12)

$$
\begin{aligned}
\int_{H_{1}}\left\langle Y, g E_{0}\right\rangle^{m}\left\langle\overline{h_{\theta} Y, g E_{0}}\right\rangle^{n} d \mu_{1}(g) & =\int_{\Sigma}\langle Y, Z\rangle^{m}\left\langle\overline{h_{\theta} Y, Z}\right\rangle^{n} d \mu_{\Sigma}(Z) \\
& =\frac{n ! \Gamma(2 p)}{\Gamma(n+2 p)} \delta_{m, n}\left\langle Y, h_{\theta} Y\right\rangle^{n} .
\end{aligned}
$$

From (2.4), (2.22) and (2.23) we have 


$$
\begin{aligned}
G_{r}\left(X_{\theta}\right) & =\frac{n ! \Gamma(2 p)}{\Gamma(n+2 p)} \delta_{m, n} \int_{N(r)}\left\langle h_{\theta}^{-1} Y, Y\right\rangle^{n} d \mu_{r}(Y) \\
& =\frac{n ! \Gamma(2 p)}{\Gamma(n+2 p)} \delta_{m, n} \int_{H_{2}}\left(\int_{H_{1}}\left\langle h_{\theta}^{-1} g_{1} g_{2} \widetilde{E}_{r}, g_{1} g_{2} \tilde{E}_{r}\right\rangle^{n} d \mu_{1}\left(g_{1}\right)\right) d \mu_{2}\left(g_{2}\right) \\
& =\frac{n ! \Gamma(2 p)}{\Gamma(n+2 p)} \delta_{m, n} \int_{H_{2}}\left(\int_{H_{1}}\left\langle g_{1} h_{\theta}^{-1} g_{2} \widetilde{E}_{r}, g_{1} g_{2} \widetilde{E}_{r}\right\rangle^{n} d \mu_{1}\left(g_{1}\right)\right) d \mu_{2}\left(g_{2}\right) \\
& =\frac{n ! \Gamma(2 p)}{\Gamma(n+2 p)} \delta_{m, n} \int_{H_{2}}\left(\int_{H_{1}}\left\langle h_{\theta}^{-1} g_{2} \tilde{E}_{r}, g_{2} \tilde{E}_{r}\right\rangle^{n} d \mu_{1}\left(g_{1}\right)\right) d \mu_{2}\left(g_{2}\right) \\
& =\frac{n ! \Gamma(2 p)}{\Gamma(n+2 p)} \delta_{m, n} \int_{H_{2}}\left\langle h_{\theta}^{-1} g_{2} \tilde{E}_{r}, g_{2} \widetilde{E}_{r}\right\rangle^{n} d \mu_{2}\left(g_{2}\right) .
\end{aligned}
$$

For $g_{2}=\operatorname{Ad}\left(\begin{array}{cccc}I_{p} & 0 & 0 & 0 \\ 0 & \alpha & 0 & \beta \\ 0 & 0 & I_{p} & 0 \\ 0 & -\bar{\beta} & 0 & \bar{\alpha}\end{array}\right) \in H_{2}$, we have

$$
\left\langle h_{\theta}^{-1} g_{2} \widetilde{E}_{r}, g_{2} \widetilde{E}_{r}\right\rangle=\cos \theta+i\left(|\alpha|^{2}-|\beta|^{2}\right)\left(2 r^{2}-1\right) \sin \theta .
$$

Therefore, it is valid

$$
G_{r}\left(X_{\theta}\right)=\frac{n ! \Gamma(2 p)}{\Gamma(n+2 p)} \delta_{m, n} \int_{S^{3}}\left\{\cos \theta+i\left(|\alpha|^{2}-|\beta|^{2}\right)\left(2 r^{2}-1\right) \sin \theta\right\}^{n} d \alpha d \beta,
$$

where $S^{3}=\left\{\left(\begin{array}{c}\alpha \\ \beta\end{array}\right) \in \mathbf{C}^{2} ;|\alpha|^{2}+|\beta|^{2}=1\right\}$, and $d \alpha d \beta$ is the volume element of $S^{3}$ which satisfies $\int_{S^{3}} 1 d \alpha d \beta=1$. If we put $\alpha=t e^{i \varphi}$ and $\beta=\left(1-t^{2}\right)^{1 / 2} e^{i \psi}$, we have

$$
\cos \theta+i\left(|\alpha|^{2}-|\beta|^{2}\right)\left(2 r^{2}-1\right) \sin \theta=\cos \theta+i\left(2 t^{2}-1\right)\left(2 r^{2}-1\right) \sin \theta
$$

and

$$
\begin{aligned}
& \int_{S^{3}}\left\{\cos \theta+i\left(|\alpha|^{2}-|\beta|^{2}\right)\left(2 r^{2}-1\right) \sin \theta\right\}^{n} d \alpha d \beta \\
& =\frac{1}{2} \pi^{-2} \int_{0}^{2 \pi}\left(\int_{0}^{2 \pi}\left(\int_{0}^{1}\left\{\cos \theta+i\left(2 t^{2}-1\right)\left(2 r^{2}-1\right) \sin \theta\right\}^{n} t d t\right) d \varphi\right) d \psi \\
& =2 \int_{0}^{1}\left\{\cos \theta+i\left(2 t^{2}-1\right)\left(2 r^{2}-1\right) \sin \theta\right\}^{n} t d t \\
& =\frac{1}{2} \int_{-1}^{1}\left\{\cos \theta+i\left(2 r^{2}-1\right) \rho \sin \theta\right\}^{n} d \rho
\end{aligned}
$$




$$
\begin{aligned}
= & \frac{1}{2 i(n+1)\left(2 r^{2}-1\right) \sin \theta}\left[\left\{\cos \theta+i\left(2 r^{2}-1\right) \rho \sin \theta\right\}^{n+1}\right]_{-1}^{1} \\
= & \frac{1}{2 i(n+1)\left(2 r^{2}-1\right) \sin \theta}\left(\left\{\cos \theta+i\left(2 r^{2}-1\right) \sin \theta\right\}^{n+1}\right. \\
& \left.-\left\{\cos \theta-i\left(2 r^{2}-1\right) \sin \theta\right\}^{n+1}\right) .
\end{aligned}
$$

If we put $c_{m, n}=\frac{n ! \Gamma(2 p)}{\Gamma(n+2 p)} \delta_{m, n}$, from (2.24) and (2.25) we have

$$
\begin{aligned}
& \int_{0}^{1} G_{r}\left(X_{\theta}\right) r^{4 p-5}\left(1-r^{2}\right)^{2 p-3}\left(2 r^{2}-1\right)^{2} d r \\
& =c_{m, n} \int_{0}^{1} \frac{r^{4 p-5}\left(1-r^{2}\right)^{2 p-3}\left(2 r^{2}-1\right)}{2 i(n+1) \sin \theta}\left(\left\{\cos \theta+i\left(2 r^{2}-1\right) \sin \theta\right\}^{n+1}\right. \\
& \left.\quad-\left\{\cos \theta-i\left(2 r^{2}-1\right) \sin \theta\right\}^{n+1}\right) d r .
\end{aligned}
$$

By putting $t=\left(1-r^{2}\right)^{1 / 2}$ we have

$$
\begin{aligned}
& \int_{0}^{1} r^{4 p-5}\left(1-r^{2}\right)^{2 p-3}\left(2 r^{2}-1\right)\left\{\cos \theta-i\left(2 r^{2}-1\right) \sin \theta\right\}^{n+1} d r \\
& \quad=-\int_{0}^{1} t^{4 p-5}\left(1-t^{2}\right)^{2 p-3}\left(2 t^{2}-1\right)\left\{\cos \theta+i\left(2 t^{2}-1\right) \sin \theta\right\}^{n+1} d t .
\end{aligned}
$$

Hence, we have

$$
\begin{aligned}
& \int_{0}^{1} G_{r}\left(X_{\theta}\right) r^{4 p-5}\left(1-r^{2}\right)^{2 p-3}\left(2 r^{2}-1\right)^{2} d r \\
= & c_{m, n} \int_{0}^{1} \frac{r^{4 p-5}\left(1-r^{2}\right)^{2 p-3}\left(2 r^{2}-1\right)}{i(n+1) \sin \theta}\left\{\cos \theta+i\left(2 r^{2}-1\right) \sin \theta\right\}^{n+1} d r \\
= & c_{m, n} \int_{0}^{1} \frac{\left\{-r^{4 p-4}\left(1-r^{2}\right)^{2 p-2}\right\}^{\prime}}{i(n+1)(4 p-4) \sin \theta}\left\{\cos \theta+i\left(2 r^{2}-1\right) \sin \theta\right\}^{n+1} d r \\
= & c_{m, n}\left[\frac{-r^{4 p-4}\left(1-r^{2}\right)^{2 p-2}}{i(n+1)(4 p-4) \sin \theta}\left\{\cos \theta+i\left(2 r^{2}-1\right) \sin \theta\right\}^{n+1}\right]_{0}^{1} \\
& +c_{m, n} \int_{0}^{1} \frac{r^{4 p-3}\left(1-r^{2}\right)^{2 p-2}}{p-1}\left\{\cos \theta+i\left(2 r^{2}-1\right) \sin \theta\right\}^{n} d r \\
= & c_{m, n} \int_{0}^{1} \frac{r^{4 p-3}\left(1-r^{2}\right)^{2 p-2}}{p-1}\left\{\cos \theta+i\left(2 r^{2}-1\right) \sin \theta\right\}^{n} d r \\
= & \frac{\pi^{1 / 2} \Gamma(2 p-2)}{2^{4 p-3} \Gamma\left(2 p-\frac{1}{2}\right)} c_{m, n} P_{n, 4 p}(\cos \theta)
\end{aligned}
$$


from (2.10). Because $P\left(X_{\theta}\right)=4$ and $\left\langle X_{\theta}, E_{0}\right\rangle=2 \cos \theta$, we have

$$
Q_{n, p}\left(X_{\theta}, E_{0}\right)=P_{n, 4 p}(\cos \theta) \text {. }
$$

Therefore, from (2.5) and (2.26) we have

$$
\begin{aligned}
F\left(X_{\theta}\right) & =\int_{0}^{1} \rho(r)\left(\int_{N(r)}\left\langle Y, E_{0}\right\rangle^{m}\left\langle\overline{Y, X_{\theta}}\right\rangle^{n} d \mu_{r}(Y)\right) d r \\
& =\int_{0}^{1} \rho(r) G_{r}\left(X_{\theta}\right) d r \\
& =\frac{n ! \Gamma(2 p)}{\Gamma(n+2 p)} \delta_{m, n} Q_{n, p}\left(X_{\theta}, E_{0}\right) .
\end{aligned}
$$

On the other hand, from (2.21) we have

$$
F\left(X_{\theta}\right)=\sum_{\substack{0 \leq l \leq n \\ l \equiv n(\bmod 2)}} C_{l,(n-l) / 2}(2 \cos \theta)^{l}
$$

because $x^{\prime}=y^{\prime}=z^{\prime}=w^{\prime}=z_{1}=w_{1}=0$ and $x_{1}=e^{i \theta}, y_{1}=e^{-i \theta}$. Since $F \in \mathscr{H}_{n}$, we have from (2.21) and the definition of $P(D)$

$$
\begin{aligned}
P(D) F(X)= & \sum_{\substack{(l, r) \in A \\
0 \leq l+2 r \leq n-2}}\left\{(n-l-2 r)(n-l-2 r+4 p-6) C_{l, r}\right. \\
+ & \left.4 C_{l+2, r}(l+2)(l+1)+4 C_{l, r+1}(l+r+2)(r+1)\right\}\left(x_{1}+y_{1}\right)^{l} \\
& \cdot\left(x_{1} y_{1}+z_{1} w_{1}\right)^{r}\left(x^{\prime} \cdot y^{\prime}+z^{\prime} \cdot w^{\prime}\right)^{(n-l-2 r-2) / 2}=0 .
\end{aligned}
$$

If $(l, r) \in \Lambda$ and $0 \leq l+2 r \leq n-2$, we have by this equation

$$
\begin{aligned}
& (n-l-2 r)(n-l-2 r+4 p-6) C_{l, r} \\
& \quad=-4 C_{l+2, r}(l+2)(l+1)-4 C_{l, r+1}(l+r+2)(r+1) .
\end{aligned}
$$

(2.28) shows that we can determine all coefficients of $F(X)$ uniquely by $C_{l,(n-l) / 2}$ $(l=n-2[n / 2], n-2[n / 2]+2, \cdots, n-2, n)$. If we put $H(X)=\frac{n ! \Gamma(2 p)}{\Gamma(n+2 p)} Q_{n, p}\left(X, E_{0}\right)$, then $H$ belongs to $\mathscr{H}_{n}$ and $H(k X)=H(X)$ for any $k \in K_{0}$ because $P(X)$ is $K$-invariant. Hence we can express

$$
H(X)=\sum_{(l, r) \in \Lambda} D_{l, r}\left(x_{1}+y_{1}\right)^{l}\left(x_{1} y_{1}+z_{1} w_{1}\right)^{r}\left(x^{\prime} \cdot y^{\prime}+z^{\prime} \cdot w^{\prime}\right)^{(n-l-2 r) / 2},
$$

where $D_{l, r} \in \mathbf{C}$. In addition, $D_{l, r}$ also satisfies (2.28). In the case $n=m$, since $H\left(X_{\theta}\right)=F\left(X_{\theta}\right)$ and 


$$
H\left(X_{\theta}\right)=\sum_{\substack{0 \leq l \leq n \\ l \equiv n(\bmod 2)}} D_{l,(n-l) / 2}(2 \cos \theta)^{l},
$$

we have $D_{l,(n-l) / 2}=C_{l,(n-l) / 2}$ by (2.27). Therefore, for any $(l, r) \in \Lambda$ we obtain $D_{l, r}=C_{l, r}$, which implies (2.20).

When $n \neq m$, we have $C_{l,(n-l) / 2}=0 \quad(l=n-2[n / 2], n-2[n / 2]+2, \cdots, n-2, n)$ because $F\left(X_{\theta}\right)=0$. Therefore, we get $F(X)=0$ by (2.28).

Q.E.D.

Proof of Theorem 2.2. (i) We get (2.6) easily from (1.1).

(ii) From Lemma 2.6 we have for any $X \in \Sigma$

$$
\begin{gathered}
\int_{0}^{1} \rho(r)\left(\int_{N(r)}\left\langle Z, E_{0}\right\rangle^{m}\langle\overline{Z, X}\rangle^{n} d \mu_{r}(Z)\right) d r \\
=\frac{n ! \Gamma(2 p)}{\Gamma(n+2 p)} \delta_{m, n} P_{n, 4 p}\left(\frac{1}{2}\left\langle X, E_{0}\right\rangle\right)
\end{gathered}
$$

For any $Y \in \Sigma$ there exists some $g \in K_{\mathbf{R}}$ such that $Y=g E_{0}$. Hence, we have

$$
\begin{aligned}
& \int_{0}^{1} \rho(r)\left(\int_{N(r)}\langle Z, Y\rangle^{m}\langle\overline{Z, X}\rangle^{n} d \mu_{r}(Z)\right) d r \\
& =\int_{0}^{1} \rho(r)\left(\int_{N(r)}\left\langle Z, g E_{0}\right\rangle^{m}\langle\overline{Z, X}\rangle^{n} d \mu_{r}(Z)\right) d r \\
& =\int_{0}^{1} \rho(r)\left(\int_{N(r)}\left\langle g^{-1} Z, E_{0}\right\rangle^{m}\left\langle\overline{g^{-1} Z, g^{-1} X}\right\rangle^{n} d \mu_{r}(Z)\right) d r \\
& =\int_{0}^{1} \rho(r)\left(\int_{N(r)}\left\langle Z, E_{0}\right\rangle^{m}\left\langle\overline{Z, g^{-1} X}\right\rangle^{n} d \mu_{r}(Z)\right) d r \\
& =\frac{n ! \Gamma(2 p)}{\Gamma(n+2 p)} \delta_{m, n} P_{n, 4 p}\left(\frac{1}{2}\left\langle g^{-1} X, E_{0}\right\rangle\right) \\
& =\frac{n ! \Gamma(2 p)}{\Gamma(n+2 p)} \delta_{m, n} P_{n, 4 p}\left(\frac{1}{2}\langle X, Y\rangle\right) \\
& =\frac{n ! \Gamma(2 p)}{\Gamma(n+2 p)} \delta_{m, n} Q_{n, p}(X, Y) .
\end{aligned}
$$

It is clear that the restriction mapping $\left.f \rightarrow f\right|_{\Sigma}$ is the bijection from $\mathscr{H}_{n}$ onto $\left.\mathscr{H}_{n}\right|_{\Sigma}$. Since the left-hand side of (2.29) and $(n ! \Gamma(2 p) / \Gamma(n+2 p)) \delta_{m, n} Q_{n, p}(X, Y)$ belong to $\mathscr{H}_{n}$, we obtain (2.7) from (2.29).

(iii) For any $f \in \mathscr{H}_{m}$ there exist some positive integer $M, a_{k} \in \mathbf{C}$ and $Y_{k} \in \Sigma$ $(k=1,2, \cdots, M)$ such that 


$$
f(Z)=\sum_{k=1}^{M} a_{k} Q_{m, p}\left(Z, Y_{k}\right) \quad(Z \in \mathfrak{p})
$$

(2.30) implies

$$
\begin{gathered}
\int_{0}^{1} \rho(r)\left(\int_{N(r)} f(Z)\langle\overline{Z, X}\rangle^{n} d \mu_{r}(Z)\right) d r \\
=\sum_{k=1}^{M} a_{k} \int_{0}^{1} \rho(r)\left(\int_{N(r)} Q_{m, p}\left(Z, Y_{k}\right)\langle\overline{Z, X}\rangle^{n} d \mu_{r}(Z)\right) d r \quad(X \in \mathfrak{p}) .
\end{gathered}
$$

Let $C_{n}$ be the coefficient of the highest power of $t$ in $P_{n, 4 p}(t)$. Then it is known that

$$
C_{n}=\frac{2^{n} \Gamma(n+2 p)}{n ! \Gamma(2 p) \operatorname{dim} \mathscr{H}_{n}}
$$

(cf. [5], [7]) and $Q_{m, p}\left(Z, Y_{k}\right)=2^{-m} C_{m}\left\langle Z, Y_{k}\right\rangle^{m}$ for $Z \in N(r) \subset \mathfrak{N}$. Hence, the right-hand side of (2.31) equals

$$
\begin{aligned}
2^{-m} C_{m} & \sum_{k=1}^{M} a_{k} \int_{0}^{1} \rho(r)\left(\int_{N(r)}\left\langle Z, Y_{k}\right\rangle^{m}\langle\overline{Z, X}\rangle^{n} d \mu_{r}(Z)\right) d r \\
= & 2^{-n} C_{n} \sum_{k=1}^{M} a_{k} \frac{n ! \Gamma(2 p)}{\Gamma(n+2 p)} \delta_{m, n} Q_{n, p}\left(X, Y_{k}\right) \\
= & \left(\operatorname{dim} \mathscr{H}_{n}\right)^{-1} \delta_{m, n} f(X)
\end{aligned}
$$

by (2.7), (2.30) and (2.32).

(iv) For any $g \in \mathscr{H}_{n}$ there exist some positive integer $M, X_{k} \in \Sigma$ and $a_{k} \in \mathbf{C}$ $(k=1,2, \cdots, M)$ such that $g(Z)=\sum_{k=1}^{M} a_{k} Q_{n, p}\left(Z, X_{k}\right)$. Then we have $g(Z)=$ $2^{-n} C_{n} \sum_{k=1}^{M} a_{k}\left\langle Z, X_{k}\right\rangle^{n}$ for $Z \in \mathfrak{N}$. Hence, from (2.6) and (2.8) we get

$$
\begin{aligned}
\int_{0}^{1} \rho(r)\left(\int_{N(r)} f(Z) \overline{g(Z)} d \mu_{r}(Z)\right) d r & =2^{-n} C_{n}\left(\operatorname{dim} \mathscr{H}_{n}\right)^{-1} \delta_{m, n} \sum_{k=1}^{M} \bar{a}_{k} f\left(X_{k}\right) \\
& =2^{-n} C_{n} \int_{\Sigma} f(Z) \overline{g(Z)} d \mu_{\Sigma}(Z)
\end{aligned}
$$

because $Q_{n, p}\left(Z, X_{k}\right)$ is real valued on $\Sigma$. Therefore, (2.9) follows from (2.32) and (2.33).

Q.E.D.

\section{References}

[1] S. Helgason, Differential Geometry, Lie Groups, and Symmetric spaces, Academic Press (1978).

[2] K. II, On a Bargmann-type transform and a Hilbert space of holomorphic functions, Tôhoku Math. J. 38 (1986), 57-69. 
[3] B. Kostant and S. Rallis, Orbits and representations associated with symmetric spaces, Amer. J. Math. 93 (1971), 753-809.

[4] M. Morimoto, Analytic functionals on the Lie sphere, Tokyo J. Math. 3 (1980), 1-35.

[5] M. Morimoto, Hyperfunctions on the Sphere, Sophia Kokyuroku in Math. 12 (1982), Dept. of Math. Sophia Univ. (in Japanese).

[6] M. Morimoto, Analytic functionals on the sphere and their Fourier-Borel transformations, Complex Analysis, Banach Center Publ. 11 (1983), PWN-Polish Scientific Publishers, 223-250.

[7] C. Müller, Spherical Harmonics, Lecture Notes in Math. 17 (1966), Springer.

[8] H. S. ShapIRo, An algebraic theorem of E. Fischer, and the holomorphic Goursat problem, Bull. London Math. Soc. 21 (1989), 513-537.

[9] A. Terras, Harmonic Analysis on Symmetric Spaces and Applications II, Springer (1988).

[10] R. WADA, On the Fourier-Borel transformations of analytic functionals on the complex sphere, Tôhoku Math. J. 38 (1986), 417-432.

[11] R. WADA, A uniqueness set for linear partial differential operators of the second order, Funkcial. Ekvac. 31 (1988), 241-248.

[12] R. WADA, Holomorphic functions on the complex sphere, Tokyo J. Math. 11 (1988), $205-218$.

[13] R. WADA, A uniqueness set for linear partial differential operators with real coefficients, Proc. Japan Acad. Ser. A 66 (1990), 278-280.

[14] R. WAdA, The integral representations of harmonic polynomials in the case of $\mathfrak{s u}(p, 1)$, Tokyo J. Math. 21 (1998), 233-245.

[15] R. WADA and A. KowATA, Holomorphic functions on the nilpotent subvariety of symmetric spaces, Hiroshima Math. J. 21 (1991), 263-266.

[16] R. WADA and M. Morimoto, A uniqueness set for the differential operator $\Delta_{z}+\lambda^{2}$, Tokyo J. Math. 10 (1987), 93-105. 